\title{
ON A METHOD IN SCATTERING THEORY
}

\author{
ì. R. AKCHURIN AND R. A. MINLOS
}

\begin{abstract}
We use the well-studied Friedrichs model to showcase a new method for proving the asymptotic completeness of two operators, which in our case are the Friedrichs operator $A$ and the operator obtained from $A$ by omitting the integral term. Technically, the problem is reduced to a detailed analysis of the Fredholm determinant and minor of an auxiliary integral operator.
\end{abstract}

\section{INTRODUCTION AND STATEMENT OF THE PROBLEM}

In the paper [1, the authors demonstrated a new method for proving the completeness of the system of the following two operators in the Hilbert space $L_{2}\left(\mathbb{R}^{d}\right), d \geq 3$ :

$$
(A f)(q)=\omega(q) f(q)+\int_{\mathbb{R}^{d}} D(q, p) f(p) d p
$$

(the Friedrichs model; see [2] and [3]) and

$$
\left(A_{0} f\right)(q)=\omega(q) f(q), \quad q \in \mathbb{R}^{d}, \quad f \in L_{2}\left(\mathbb{R}^{d}\right) .
$$

Here $\omega$ is a smooth real-valued function increasing to infinity as $q \rightarrow \infty$ and having a unique critical point, namely, a nondegenerate minimum at the point $q_{0}=0$. The kernel $D$ belongs to the space $S\left(\mathbb{R}^{d} \times \mathbb{R}^{d}\right)$ (see [4] and [5]) and satisfies the condition

$$
D(q, p)=\overline{D(p, q)},
$$

which ensures that $A$ is selfadjoint.

It was assumed in 1 that $D$ is sufficiently small, which ensures that $A$ has no eigenvalues. Here we drop this assumption and hence consider the case in which there exist eigenvalues of $A$. We only assume that all of them lie outside the continuous spectrum of $A$. Apart from this, it is known that $A$ has only finitely many eigenvalues, all of them are of finite multiplicity, and the singular spectrum of $A$ is empty (see [3]).

Let $\left\{\varphi_{j}\right\}_{j=1}^{k}$ be the orthonormal system formed by all eigenfunctions of $A$, and let $\mathcal{H}=\left\{\varphi_{j}\right\}^{\perp}$ be the orthogonal complement of this system. Obviously, $\mathcal{H}$ is an invariant subspace for $A$, and we denote the restriction of $A$ to $\mathcal{H}$ by $\widetilde{A}$. The following theorem is the main result of this paper.

Theorem. The operators $\widetilde{A}$ and $A_{0}$ are unitarily equivalent.

Remark. The fact stated in the theorem is well known (see [2] and [3]). We use its proof as an example to present our method. The method also applies to more complicated cases (see [13]).

2010 Mathematics Subject Classification. Primary 47A40; Secondary 35P25, 35Q40.

Key words and phrases. Asymptotic completeness, Friedrichs model, wave operators, Fredholm minor, Fredholm determinant, stationary phase method. 
The theorem follows [6] from the existence of the following strong limits (known as wave operators):

$$
\begin{array}{ll}
W f={\underset{t \rightarrow+\infty}{\mathrm{s}-\lim } e^{i t A} e^{-i t A_{0}} f,} f \in L_{2}\left(\mathbb{R}^{d}\right) & \text { (the direct wave operator), } \\
\widehat{W} g={\underset{t \rightarrow+\infty}{\mathrm{s}-\lim } e^{i t A_{0}} e^{-i t A} g, \quad g \in \mathcal{H}} & \text { (the inverse wave operator). }
\end{array}
$$

If the operators $W$ and $\widehat{W}$ exist, then they are inverses of each other,

$$
W L_{2}\left(\mathbb{R}^{d}\right)=\mathcal{H}, \quad \widehat{W} \mathcal{H}=L_{2}\left(\mathbb{R}^{d}\right), \quad \text { and } \quad \widehat{W}=W^{-1},
$$

and define unitary mappings of the spaces $L_{2}\left(\mathbb{R}^{d}\right)$ and $\mathcal{H}$ onto each other, taking the operator $A_{0}$ to $\widetilde{A}$,

$$
\widetilde{A}=W A_{0} \widehat{W} .
$$

The existence of both limits (1.3a) and (1.3b) is established here by Cook's method (see [6]). Namely, one has

$$
\int_{0}^{\infty}\left\|V e^{-i t A_{0}} f\right\|_{L_{2}\left(\mathbb{R}^{d}\right)} d t<\infty
$$

where $V=A-A_{0}$, for all functions $f \in S\left(\mathbb{R}^{d}\right)$, which obviously form an everywhere dense set in $L_{2}\left(\mathbb{R}^{d}\right)$. The existence of the limit (1.3a) follows from (1.6) (see [6]). We shall show that

$$
\int_{0}^{\infty}\left\|V e^{-i t A} g\right\|_{L_{2}\left(\mathbb{R}^{d}\right)} d t<\infty
$$

for all $g \in S\left(\mathbb{R}^{d}\right) \cap \mathcal{H}$. (This set is everywhere dense in $\mathcal{H}$, because the eigenfunctions $\varphi_{j}$ of $A$ lie in $S\left(\mathbb{R}^{d}\right)$.) The existence of the limit (1.3b) follows from (1.7).

Inequality (1.6) was proved in [1] with the use of the stationary phase method; this proof does not assume that $D$ is small, and so it applies in our case. Hence we proceed to the proof on inequality (1.7).

Let us represent the vector $e^{-i t A} g$ in the form

$$
e^{-i t A} g=\frac{1}{2 \pi i} \int_{\gamma} e^{-i t z} R_{A}(z) g d z,
$$

where $R_{A}(z)=(A-z E)^{-1}$ is the resolvent of $A$ and the integral is taken over a contour $\gamma \subset \mathbb{C}^{1}$ surrounding the spectrum of $A$ clockwise (see [7]).

\section{The Resolvent $R_{A}(z)$ And Related estimates}

Let $\Sigma=\left\{\lambda_{j}\right\}$ be the set of eigenvalues of $A$. For each $z \in \mathbb{C}^{1} \backslash([\varkappa, \infty) \cup \Sigma)$, where $\varkappa=\min _{q} \omega(q)$, the action of $R_{A}(z)$ on a vector $g \in L_{2}\left(\mathbb{R}^{d}\right)$ can be represented in the form

$$
\left(R_{A}(z) g\right)(q)=\frac{g(q)}{\omega(q)-z}+\Delta^{-1}(z) \int_{\mathbb{R}^{d}} \frac{T(q, p ; z) g(p) d p}{(\omega(q)-z)(\omega(p)-z)} .
$$

The function $T(q, p ; z)$ (the Fredholm minor [8]) is given by

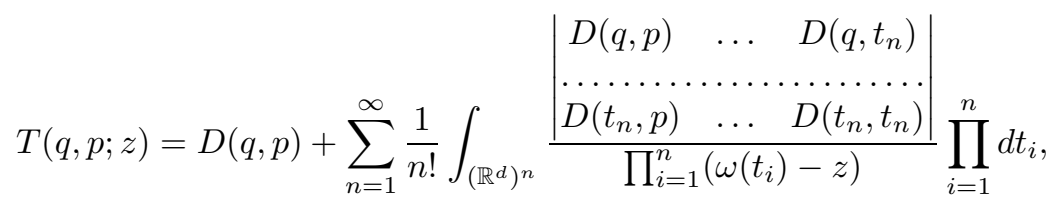


and the function $\Delta(z)$ (the Fredholm determinant [8]) has the form

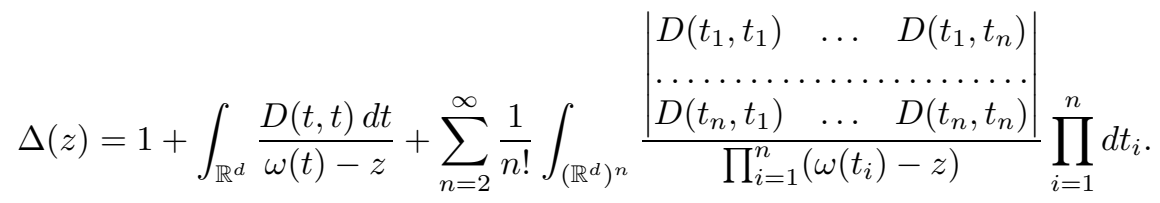

Since the first term in (2.1) is equal to $\left(R_{A_{0}}(z) g\right)(q)$ and the operator $e^{-i t A_{0}}$ admits a representation similar to (1.8), we see, by virtue of (1.6), that it suffices to prove that the function

$$
\begin{aligned}
\| V\left(e^{-i t A}-e^{-i t A_{0}}\right) & g \|_{L_{2}\left(\mathbb{R}^{d}\right)} \\
& =\left\|\int_{\gamma} d z e^{-i t z} \frac{1}{\Delta(z)} \int_{\left(\mathbb{R}^{d}\right)^{2}} \frac{D(q, p) T(p, s ; z) g(s)}{(\omega(p)-z)(\omega(s)-z)} d p d s\right\|_{L_{2}\left(\mathbb{R}^{d}\right)}
\end{aligned}
$$

of the variable $t$ is integrable with respect to $t$ on $(0, \infty)$ for $g \in S\left(\mathbb{R}^{d}\right) \cap \mathcal{H}$. For brevity, the right-hand side of (2.4) can be written in the form

$$
\left\|\int_{\gamma} d z e^{-i t z} \frac{1}{\Delta(z)} \sum_{n=0}^{\infty} \frac{1}{n !} I_{n}(\cdot, z)\right\|_{L_{2}\left(\mathbb{R}^{d}\right)},
$$

where

$$
I_{n}(q, z)=\int_{\left(\mathbb{R}^{d}\right)^{n+2}} \frac{K_{n}\left(q, p, s, t_{1}, \ldots, t_{n}\right)}{(\omega(p)-z)(\omega(s)-z) \prod_{j=1}^{n}\left(\omega\left(t_{j}\right)-z\right)} d p d s \prod_{j=1}^{n} d t_{j}
$$

here

$$
K_{n}\left(q, p, s,\left\{t_{j}\right\}\right)=D(q, p) g(s)\left|\begin{array}{ccc}
D(p, s) & \ldots & D\left(p, t_{n}\right) \\
\ldots \ldots \ldots & \ldots & \ldots \ldots \ldots \\
D\left(t_{n}, s\right) & \ldots & D\left(t_{n}, t_{n}\right)
\end{array}\right| \text { for } n>0
$$

and

$$
K_{0}(q, p, s)=D(q, p) D(p, s) g(s) .
$$

Note that $K_{n}\left(q, p, s, t_{1}, \ldots, t_{n}\right)$ belongs to the space $S\left(\mathbb{R}^{d(n+3)}\right)$ of rapidly decaying functions of $n+3$ variables. By using Hadamard's inequality [9], we obtain the following estimate for $K_{n}$ :

$$
\left|K_{n}\left(q, p, s,\left\{t_{j}\right\}_{j=1}^{n}\right)\right| \leq \frac{C L^{n} n^{n / 2}}{\left(q^{2}+1\right)^{d}\left(p^{2}+1\right)^{d}\left(s^{2}+1\right)^{d} \prod_{j=1}^{n}\left(t_{j}^{2}+1\right)^{d}}
$$

with constants $C=C(g)>0$ and $L=L(D)>0$. Similar estimates hold for the derivatives of $K_{n}$.

Note that, for any $n$ and $q$, the function $I_{n}(q, z)$, which is defined for all complex $z$ outside the cut $[\varkappa, \infty)$, has the limits

$$
I_{n}^{ \pm}(q, x)=\lim _{\varepsilon \downarrow 0} I_{n}(q, x \pm i \varepsilon), \quad-\infty<x<\infty,
$$

as $z$ approaches the real axis from above $(+)$ and below $(-)$, and these limits are uniform with respect to $n$ and $q$ (e.g., see [10] and [11]). Obviously, the two limits coincide for $x<\varkappa$.

Furthermore, it follows from (2.7) and the smoothness of $K_{n}$ that the estimate

$$
\left|I_{n}^{ \pm}(q, x)\right| \leq \frac{\bar{C} n^{n / 2} M^{n}}{\left(q^{2}+1\right)^{d}}
$$


holds with some constants $\bar{C}>0$ and $M>0$. It follows from (2.8) that the series

$$
I^{ \pm}(q, x)=\sum_{n=0}^{\infty} \frac{1}{n !} I_{n}^{ \pm}(q, x)
$$

converges. It is convenient to represent the function $I^{ \pm}(q, x)$ in the form

$$
I^{ \pm}(q, x)=\sum_{n=0}^{\infty} \frac{1}{n !} \int_{\left(\mathbb{R}^{d}\right)^{n+2}} \frac{K_{n}\left(q, p, s,\left\{t_{j}\right\}_{j=1}^{n}\right) d p d s \prod_{j=1}^{n} d t_{j}}{(\omega(p)-x \mp i 0)(\omega(s)-x \mp i 0) \prod_{j=1}^{n}\left(\omega\left(t_{j}\right)-x \mp i 0\right)}
$$

By analogy with the preceding, the function $\Delta(z)$ has limits

$$
\Delta^{ \pm}(x)=\lim _{\varepsilon \downarrow 0} \Delta(x \pm i \varepsilon)
$$

which, by analogy with (2.10), can be represented in the form

$$
\Delta^{ \pm}(x)=1+\sum_{n=1}^{\infty} \frac{1}{n !} \int_{\left(\mathbb{R}^{d}\right)^{n}} \frac{G_{n}\left(s_{1}, \ldots, s_{n}\right)}{\prod_{j=1}^{n}\left(\omega\left(s_{j}\right)-x \mp i 0\right)} \prod_{j=1}^{n} d s_{j}=1+J^{ \pm}(x),
$$

where the function $G_{n}$ coincides with the corresponding determinant in (2.3). Clearly, $G_{n} \in S\left(\left(\mathbb{R}^{d}\right)^{n}\right)$ and

$$
\left|G_{n}\left(s_{1}, \ldots, s_{n}\right)\right| \leq \frac{n^{n / 2} \bar{M}^{n}}{\prod_{j=1}^{n}\left(s_{j}^{2}+1\right)^{d}}
$$

by Hadamard's inequality. Similar estimates hold for the derivatives of $G_{n}$.

For the contour $\gamma$ in (1.8) we take two lines $l_{ \pm}$, where $l_{+}$coincides with the real ray $(-\infty, \varkappa)$ and then with the upper bank of the cut $[\varkappa, \infty)$, while $l_{-}$coincides with the same ray and then with the lower bank of the cut.

Thus, for this choice of $\gamma$, the expression whose norm is taken in (2.4) can be represented in the form

$$
\int_{l_{+}} e^{-i t x} \frac{I^{+}(q, x)}{\Delta^{+}(x)} d x+\int_{l_{-}} e^{-i t x} \frac{I^{-}(q, x)}{\Delta^{-}(x)} d x .
$$

Next, note that the second term in (2.14) is zero for $t>0$. Indeed, if $t>0$, then the function

$$
e^{-i t z} \frac{I(q, z)}{\Delta(z)}
$$

analytic in the lower half-plane, tends to zero as $z \rightarrow \infty$; hence, by shifting the contour $l_{-}$down along the imaginary axis, we can make the integral of the function (2.15) along the shifted contour as small as desired.

Thus, we have reduced the problem to estimating the first integral in (2.14). First, let us separately estimate the integrals of the numerator and denominator of this fraction.

Lemma 1. One has the estimates

$$
\begin{aligned}
& \left|\int_{l_{+}} e^{-i t x} I^{+}(q, x) d x\right| \leq \frac{C l(t)}{\left(q^{2}+1\right)^{d}}, \\
& \left|\int_{l_{+}} e^{-i t x} J^{+}(x) d x\right| \leq l(t),
\end{aligned}
$$

where the function $l(t) \geq 0(l(t)=0$ for $t<0)$ is integrable on $(0, \infty)$ and $C>0$ is some constant. 
Proof. First, consider inequality (2.16b). Obviously, the integral can be expanded in a series whose $n$th term can be represented in the form

$$
\int_{-\infty}^{\infty} d x \int_{\left(\mathbb{R}^{d}\right)^{n} \times\left(\mathbb{R}^{1}\right)^{n}} \prod_{j} d s_{j} \prod_{j} d x_{j} \frac{G_{n}\left(s_{1}, \ldots, s_{n}\right) \prod_{j} e^{-i t_{j} x_{j}}}{\prod_{j}\left(\omega\left(s_{j}\right)-x_{j}-i 0\right)} \prod_{j} \delta\left(x-x_{j}\right),
$$

where $t_{1}>0, \ldots, t_{n}>0$ are numbers such that $\sum_{j=1}^{n} t_{j}=t$.

It is easy to compute the integral (2.17); the result (see Appendix A) is

$$
2 \pi \int_{\left\{\tau_{1}>0, \ldots, \tau_{n}>0, \tau_{1}+\cdots+\tau_{n}=t\right\}} S_{n}\left(\tau_{1}, \ldots, \tau_{n}\right) \prod_{j=1}^{n} d \tau_{j},
$$

where

$$
S_{n}\left(\tau_{1}, \ldots, \tau_{n}\right)=\prod_{j=1}^{n}\left(2 \pi i \theta\left(\tau_{j}\right)\right) \int_{\left(\mathbb{R}^{d}\right)^{n}} G_{n}\left(s_{1}, \ldots, s_{n}\right) \prod_{j=1}^{n} e^{-i \tau_{j} \omega\left(s_{j}\right)} \prod_{j=1}^{n} d s_{j} .
$$

(Here $\theta(\tau)=1$ for $\tau>0$ and $\theta(\tau)=0$ for $\tau \leq 0$.) By using the stationary phase method [12] and Hadamard's inequality, we find that

$$
\left|S_{n}\left(\tau_{1}, \ldots, \tau_{n}\right)\right| \leq n^{n / 2} \bar{C}^{n} \prod_{j=1}^{n} \theta\left(\tau_{j}\right) \prod_{j=1}^{n} \frac{1}{\left(\tau_{j}+1\right)^{d / 2}},
$$

where $\bar{C}>0$ is some constant. A detailed derivation of this estimate is given in Appendix B. By setting

$$
l(t)=\sum_{n=1}^{\infty} \frac{n^{n / 2}}{n !} \bar{C}^{n} \int_{\left\{\tau_{1}+\cdots+\tau_{n}=t\right\}} \prod_{j=1}^{n} \frac{1}{\left(\tau_{j}+1\right)^{d / 2}} \prod_{j=1}^{n} d \tau_{j}
$$

for $t>0$, we see that $l(t)$ is integrable and the estimate (2.16b) is satisfied. The estimate (2.16a) can be obtained in a similar way. The proof of Lemma 1 is complete.

\section{Derivation of inequality (1.7)}

First, consider the simplest case in which $A$ has no eigenvalues. Then, as follows from Lemma 2 below, $\Delta^{+}(x) \neq 0$ for $x<\varkappa$. Next, we state a general assumption:

If the operator $A$ has no eigenvalues lying on the continuous spectrum $[\varkappa, \infty)$, then $\Delta^{+}(x) \neq 0$ for all $x \in[\varkappa, \infty)$.

Thus, in our case, $\Delta^{+}(x) \neq 0$ for all $x \in \mathbb{R}$.

Now consider the Banach algebra $\mathcal{J}$ (see [14]) of continuous functions $f(\cdot)$ defined on the extended real line $(-\infty, \infty) \cup\{\infty\}$ and representable in the form

$$
f(x)=c+\varphi(x),
$$

where $c=f(\infty)$ is a constant and the Fourier transform $\widetilde{\varphi}(\cdot)$ of the function $\varphi(\cdot)$ is integrable,

$$
\widetilde{\varphi}(\cdot) \in L_{1}(\mathbb{R}) .
$$

The norm $\|f\|_{\mathcal{J}}$ is defined by the formula

$$
\|f\|_{\mathcal{J}}=|c|+\|\widetilde{\varphi}\|_{L_{1}(\mathbb{R})} .
$$

Next, let $\mathcal{M}$ be the following ideal in $\mathcal{J}$ :

$$
\mathcal{M}=\{f \in \mathcal{J} \mid f(\infty)=0\} .
$$

It follows from (2.16a) and (2.16b) that $I^{+}(q, \cdot) \in \mathcal{M}$ for each $q$ and that $J^{+}(\cdot) \in \mathcal{M}$. Thus, $\Delta^{+}=1+J^{+} \in \mathcal{J}$, and the function $\Delta^{+}$vanishes nowhere. By the well-known 
Wiener-Gelfand theorem (see [14]), there exists a reciprocal function $\left(\Delta^{+}\right)^{-1} \in \mathcal{J}$, and $\left(\Delta^{+}\right)^{-1}=1+\widehat{J}$, where $\widehat{J} \in \mathcal{M}$. We see that

$$
R(q, x):=\frac{I^{+}(q, x)}{\Delta^{+}(x)}=(1+\widehat{J}(x)) I^{+}(q, x)=I^{+}(q, x)+\widehat{J}(x) I^{+}(q, x) \in \mathcal{M}
$$

and the Fourier transform of $R(q, x)$ admits the estimate

$$
|\widetilde{R}(q, t)| \leq \frac{\bar{C}}{\left(q^{2}+1\right)^{d}} \cdot(l(t)+(k * l)(t)),
$$

where $k$ is the Fourier transform of $\widehat{J}$ and $*$ stands for the convolution of two functions. Since $k * l$ is integrable on $(0, \infty)$, it readily follows from (3.2) that (1.7) holds.

Now consider the general case in which $A$ has eigenvalues $\left\{\lambda_{j}\right\}_{j=1}^{k},\left|\lambda_{1}\right| \geq\left|\lambda_{2}\right| \geq$ $\cdots \geq\left|\lambda_{k}\right|$, and the corresponding eigenfunctions $\left\{\varphi_{j}\right\}_{j=1}^{k}$. Then the function $\Delta^{+}(x)$ has zeros on the half-line $(-\infty, \varkappa)$, and the ratio $R(q, x)$ may have singularities in general. However, we shall show that there are no such singularities for $g \in \mathcal{H}$. First, we prove the following lemma.

Lemma 2. A real number $\lambda_{0}<\varkappa$ is an eigenvalue of $A$ if and only if $\Delta^{+}\left(\lambda_{0}\right)=0$. The multiplicity of the zero $\lambda_{0}$ coincides with the dimension of the eigenspace $E_{\lambda_{0}} \subset L_{2}\left(\mathbb{R}^{d}\right)$ corresponding to this eigenvalue.

Proof. Suppose that $\lambda_{0} \in(-\infty, \varkappa)$ is not a zero of $\Delta^{+}(x)$. Then formula (2.1) shows that the resolvent $R_{A}\left(\lambda_{0}\right)$ is defined, and hence $\lambda_{0}$ cannot be an eigenvalue. Thus, every eigenvalue $\lambda<\varkappa$ of $A$ is a zero of $\Delta^{+}(x)$.

To prove the converse, for each real $\lambda<\varkappa$ consider two integral operators $K_{1}(\lambda)$ and $K_{2}(\lambda)$ with kernels

$$
K_{1}(q, p ; \lambda)=\frac{D(q, p)}{\omega(p)-\lambda} \quad \text { and } \quad K_{2}(q, p ; \lambda)=\frac{D(q, p)}{\sqrt{(\omega(q)-\lambda)(\omega(p)-\lambda)}},
$$

respectively. Since the function $D(q, p)$ is smooth and rapidly decays at infinity, it follows that $K_{2}(\lambda)$ is a selfadjoint trace class operator (see [15]). Let $\left\{\mu_{s}(\lambda)\right\}_{s=1}^{\infty}$ and $\left\{\psi_{s}(q ; \lambda)\right\}_{s=1}^{\infty}$ be the sequences of its eigenvalues numbered in descending order of absolute values and the corresponding normalized eigenfunctions, respectively. We use the representation

$$
K_{2}(q, p ; \lambda)=\sum_{j} \mu_{j}(\lambda) \psi_{j}(q ; \lambda) \bar{\psi}_{j}(p ; \lambda)
$$

and the fact that the operators $K_{1}(\lambda)$ and $K_{2}(\lambda)$ are similar to write out the following chain of equalities:

$$
\Delta\left(\lambda ; K_{2}(\lambda)\right)=\sum_{\left\{j_{s}\right\}} \prod_{s=1}^{k} \mu_{j_{s}}(\lambda)=\prod_{j}\left(1+\mu_{j}(\lambda)\right)=\Delta\left(\lambda ; K_{1}(\lambda)\right),
$$

where the $\Delta\left(\lambda ; K_{i}(\lambda)\right), i=1,2$, are the Fredholm determinants computed by Fredholm's well-known formulas (see [8]) for the operators $E+K_{i}(\lambda)$; as is seen from (2.3), one has $\Delta\left(\lambda ; K_{1}(\lambda)\right)=\Delta^{+}(\lambda)$ for $\lambda<\varkappa$. It follows from the representation (3.3) that the equation $\Delta^{+}\left(\lambda_{0}\right)=0$ is equivalent to the fact that one or several eigenvalues satisfy $\mu_{s}\left(\lambda_{0}\right)=-1$. Since the $\mu_{s}$ decay to zero with increasing $s$, it follows that there exist finitely many (say, $m\left(\lambda_{0}\right)$ ) such eigenvalues. It is easy to obtain

$$
\left.\mu_{s}^{\prime}(\lambda)\right|_{\lambda=\lambda_{0}}=\left.\iint \frac{d K_{2}}{d \lambda}(q, p ; \lambda)\right|_{\lambda=\lambda_{0}} \psi_{s}\left(q ; \lambda_{0}\right) \bar{\psi}_{s}\left(p ; \lambda_{0}\right) d q d p
$$


for $\lambda_{0}$ and $s$ such that $\mu_{s}\left(\lambda_{0}\right)=-1$; from this, we readily find that

$$
\mu_{s}^{\prime}\left(\lambda_{0}\right)=-\int_{\mathbb{R}^{d}} \frac{\left|\psi_{s}\left(q ; \lambda_{0}\right)\right|^{2}}{\omega(q)-\lambda_{0}} d q<0 .
$$

Thus, in a small neighborhood $U\left(\lambda_{0}\right)$ of $\lambda_{0}$ one has

$$
1+\mu_{s}(\lambda)=-C_{s}(\lambda)\left(\lambda-\lambda_{0}\right),
$$

where $C_{s}(\lambda)>0$. We see that

$$
\Delta^{+}(\lambda)=\left(\lambda-\lambda_{0}\right)^{m\left(\lambda_{0}\right)} b(\lambda)
$$

in the same neighborhood, where $b(\lambda) \neq 0$ for $\lambda \in U\left(\lambda_{0}\right)$. Finally,

$$
\Delta^{+}(\lambda)=\prod_{j}\left(\lambda-\lambda_{j}\right) v(\lambda)
$$

where $v(\lambda) \neq 0$ for any $\lambda$ and the product $\prod_{j}$ is taken over the set $\left\{\lambda_{j}\right\}$ of all zeros of $\Delta^{+}$ (with regard for their multiplicities). In what follows, we give a closed-form expression for $v(\lambda)$, which implies that $v \in \mathcal{M}$.

Next, since

$$
K_{2}\left(\lambda_{0}\right) \psi_{s}+\psi_{s}=0
$$

(for the corresponding $\psi_{s}$, whose number is equal to $m\left(\lambda_{0}\right)$ ) and

$$
K_{2}\left(q, p ; \lambda_{0}\right)=K_{1}\left(q, p ; \lambda_{0}\right) \sqrt{\frac{\omega(q)-\lambda_{0}}{\omega(p)-\lambda_{0}}},
$$

it follows that the function

$$
\varphi_{s}(q)=\frac{\psi_{s}(q)}{\sqrt{\omega(q)-\lambda_{0}}}
$$

is an eigenfunction of $A$ corresponding to the eigenvalue $\lambda_{0}$; i.e., the dimension of the eigenspace $E\left(\lambda_{0}\right)$ is $m\left(\lambda_{0}\right)$. The proof of Lemma 2 is complete.

It turns out that, for $g \in \mathcal{H}$, the function $I^{+}(q, x)$ admits an expansion similar to (3.5) as well.

Lemma 3. If the function $\Delta^{+}$has zeros $\left\{\lambda_{j}\right\}$ with $\lambda_{j}<\varkappa$, then the representations

$$
\Delta^{+}(x)=\prod_{j=1}^{k}\left(x-\lambda_{j}\right) v(x)
$$

hold and, for $g \in \mathcal{H}$, one has

$$
I^{+}(q, x)=\prod_{j=1}^{k}\left(x-\lambda_{j}\right) I^{\prime}(q, x),
$$

where $v(\cdot)$ and $I^{\prime}(q, \cdot)($ for each $q)$ belong to the ideal $\mathcal{M}$ of the algebra $\mathcal{J}$ and $v(x)$ vanishes nowhere. The Fourier transform $\widetilde{I}^{\prime}(q, t)$ admits an estimate similar to (2.16a).

Prior to proving this lemma, let us derive inequality (1.7) from it. Note that the ratio (3.1) can be represented as

$$
R(q, x)=\frac{I^{+}(q, x)}{\Delta^{+}(x)}=\frac{I^{\prime}(q, x)}{v(x)} .
$$

However, to apply the Wiener-Gelfand theorem to this ratio in the same way as above, we should multiply the numerator and denominator in (3.7) by a continuous nowhere vanishing function $H(x)$ such that the following conditions are satisfied: 
1. For each $q$, the function $W(q, x):=H(x) I^{\prime}(q, x)$ lies in $\mathcal{M}$, and its Fourier transform $\widetilde{W}(q, t)$ satisfies the estimate (2.16a).

2. The function $S(x):=H(x) v(x)$ lies in $\mathcal{J}$ and vanishes nowhere. (In particular, $S(\infty) \neq 0$.)

In this case, the above argument related to the Wiener-Gelfand theorem applies to the ratio $R(q, x)=\frac{W(q, x)}{S(x)}$, and we arrive at inequality (1.7).

Let us proceed to the construction of the function $H$. Let

$$
G(z)=\nu \int_{a}^{b} \frac{(t-a)^{4}(b-t)^{4}}{z-t} d t, \quad z \in \mathbb{C} \backslash[a, b],
$$

where $\nu>0$ is a real parameter, the interval $(a, b)$ lies to the left of $\varkappa$ and contains all zeros $\left\{\lambda_{j}\right\}$ of $\Delta^{+}$, and $a=\min _{j} \lambda_{j}-1$. Obviously, the function $G(z)$ is analytic in the domain $\mathbb{C} \backslash[a, b]$, and there exists a limit

$$
G^{+}=\lim _{\varepsilon \downarrow 0} G(x+i \varepsilon) .
$$

We readily find that $G^{+} \in \mathcal{M}$. Set

$$
H(x)=G^{+}(x)+\prod_{j=1}^{k}\left(x-\lambda_{j}\right),
$$

where the product $\prod_{j}$ is the same as in (3.6a). Let us show that $H(x) \neq 0$ for any $x$. Indeed,

1. $x \geq b \Rightarrow H(x)>0$.

2. $a<x<b \Rightarrow \operatorname{Im} H(x) \neq 0$.

3. $x \leq a$ and $k$ is odd $\Rightarrow H(x)<0$.

4. $x \leq a, k$ is even, and $\nu>0$ is sufficiently small $\Rightarrow H(x)>0$.

The function $S(x)=H(x) v(x)=G^{+}(x) v(x)+\Delta^{+}(x)$ belongs to $\mathcal{J}$ and vanishes nowhere. (In particular, $S(\infty)=1$.) Thus, $S^{-1}(x) \in \mathcal{J}$ by the Wiener-Gelfand theorem. Next, $W(q, x)=H(x) I^{\prime}(q, x)=G^{+}(x) I^{\prime}(q, x)+I^{+}(q, x) \in \mathcal{M}$ for every $q$, and the Fourier transform $\tilde{W}(q, t)$ of $W(q, x)$ satisfies the estimate (2.16a). Thus, the two conditions preceding the construction of $H$ are satisfied, and we know that these conditions imply inequality (1.7).

\section{Proof of Lemma 3}

Let $\varphi_{1}, \ldots, \varphi_{s}, s \leq k$, be an orthonormal set of eigenfunctions of $A$, and let $\lambda_{1}, \ldots, \lambda_{s}$ be the corresponding eigenvalues. We introduce the kernel

$$
L_{s}\left(t_{1}, t_{2}\right)=D\left(t_{1}, t_{2}\right)+\sum_{i=1}^{s}\left(\omega\left(t_{2}\right)-\lambda_{i}\right) \varphi_{i}\left(t_{1}\right) \bar{\varphi}_{i}\left(t_{2}\right)
$$

and note that

$$
\int_{\mathbb{R}^{d}} L_{s}\left(t_{1}, t_{2}\right) \bar{\varphi}_{i}\left(t_{1}\right) d t_{1}=0
$$

for each $i=1, \ldots, s$. 
The determinant $\Delta(z)$ admits the representation

$$
\begin{aligned}
& \Delta(z)=\prod_{j=1}^{s}\left(\lambda_{j}-z\right) \sum_{n=s}^{\infty} \frac{1}{n !} \int_{\left(\mathbb{R}^{d}\right)^{n}} \frac{\prod_{i=1}^{n} d t_{i}}{\prod_{i=1}^{n}\left(\omega\left(t_{i}\right)-z\right)}
\end{aligned}
$$

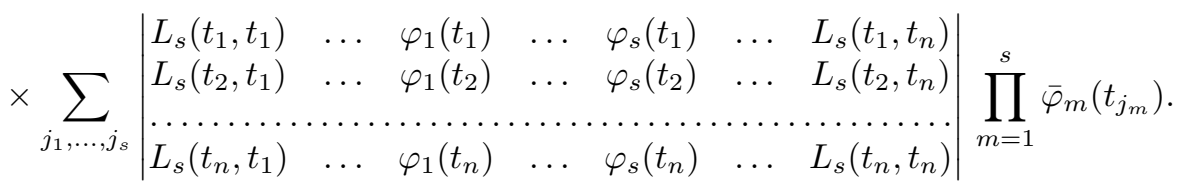

In this determinant, $s$ columns with numbers $j_{1}, \ldots, j_{s}$ have the form

$$
\left(\varphi_{m}\left(t_{1}\right), \ldots, \varphi_{m}\left(t_{n}\right)\right)^{\top}, \quad m=1, \ldots, s
$$

and the entries in the remaining columns are occupied by the kernel $L_{s}\left(t_{i}, t_{l}\right)$, where $l \neq j_{m}$ for any $m$. The inner sum $\sum_{j_{1}, \ldots, j_{s}}$ is taken over all arrangements of $s$ numbered columns in $n$ places.

Formula (4.2) can be derived by induction on $s$. We start from $s=1$. Then

$$
\begin{aligned}
& \int_{\left(\mathbb{R}^{d}\right)^{n}} \frac{\prod_{i=1}^{n} d t_{i}}{\prod_{i=1}^{n}\left(\omega\left(t_{i}\right)-z\right)}\left|\begin{array}{lll}
D\left(t_{1}, t_{1}\right) & \ldots & D\left(t_{1}, t_{n}\right) \\
\ldots \ldots \ldots \ldots \ldots & \ldots \ldots \ldots \ldots \\
D\left(t_{n}, t_{1}\right) & \ldots & D\left(t_{n}, t_{n}\right)
\end{array}\right|=\int_{\left(\mathbb{R}^{d}\right)^{n}} \frac{\prod_{i=1}^{n} d t_{i}}{\prod_{i=1}^{n}\left(\omega\left(t_{i}\right)-z\right)}
\end{aligned}
$$

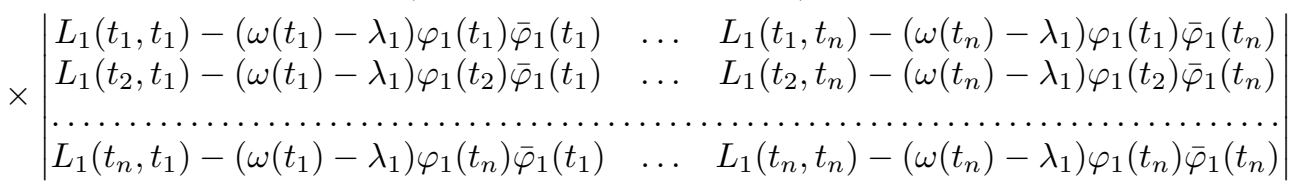

$$
\begin{aligned}
& =R_{n}-\int_{\left(\mathbb{R}^{d}\right)^{n}} \sum_{j=1}^{n} \frac{\prod_{i} d t_{i}}{\prod_{i \neq j}\left(\omega\left(t_{i}\right)-z\right)} \\
& \times\left|\begin{array}{ccccc}
L_{1}\left(t_{1}, t_{1}\right) & \ldots & \varphi_{1}\left(t_{1}\right) & \ldots & L_{1}\left(t_{1}, t_{n}\right) \\
L_{1}\left(t_{2}, t_{1}\right) & \ldots & \varphi_{1}\left(t_{2}\right) & \ldots & L_{1}\left(t_{2}, t_{n}\right) \\
\ldots \ldots \ldots \ldots \ldots & \ldots \ldots \ldots \ldots & \ldots \ldots \\
\ldots L_{1}\left(t_{n}, t_{1}\right) & \ldots & \varphi_{1}\left(t_{n}\right) & \ldots & L_{1}\left(t_{n}, t_{n}\right)
\end{array}\right| \frac{\left(\omega\left(t_{j}\right)-\lambda_{1}\right) \bar{\varphi}_{1}\left(t_{j}\right)}{\omega\left(t_{j}\right)-z} .
\end{aligned}
$$

In the last determinant, all columns but the $j$ th are occupied by $L_{1}$, and $R_{n}$ stands for the integral

$$
R_{n}=\int_{\left(\mathbb{R}^{d}\right)^{n}} \frac{\prod_{i=1}^{n} d t_{i}}{\prod_{i=1}^{n}\left(\omega\left(t_{i}\right)-z\right)}\left|\begin{array}{lll}
L_{1}\left(t_{1}, t_{1}\right) & \ldots & L_{1}\left(t_{1}, t_{n}\right) \\
\ldots \ldots \ldots \ldots & \ldots & \ldots \ldots \ldots \\
L_{1}\left(t_{n}, t_{1}\right) & \ldots & L_{1}\left(t_{n}, t_{n}\right)
\end{array}\right|
$$

We represent the last factor in (4.3) in the form

$$
\frac{\left(\omega\left(t_{j}\right)-\lambda_{1}\right) \bar{\varphi}_{1}\left(t_{j}\right)}{\omega\left(t_{j}\right)-z}=\left(1+\frac{z-\lambda_{1}}{\omega\left(t_{j}\right)-z}\right) \bar{\varphi}_{1}\left(t_{j}\right)
$$


and write out each term of the sum on the right-hand side in (4.3) in the form of two summands,

$$
\begin{aligned}
& \left(z-\lambda_{1}\right) \int_{\left(\mathbb{R}^{d}\right)^{n}} \frac{\prod_{i} d t_{i}}{\prod_{i}\left(\omega\left(t_{i}\right)-z\right)}\left|\begin{array}{lllll}
L_{1}\left(t_{1}, t_{1}\right) & \ldots & \varphi_{1}\left(t_{1}\right) & \ldots & L_{1}\left(t_{1}, t_{n}\right) \\
L_{1}\left(t_{2}, t_{1}\right) & \ldots & \varphi_{1}\left(t_{2}\right) & \ldots & L_{1}\left(t_{2}, t_{n}\right) \\
\ldots \ldots \ldots \ldots \ldots \ldots . \ldots \ldots \ldots & \ldots \ldots \ldots \ldots \ldots \\
L_{1}\left(t_{n}, t_{1}\right) & \ldots & \varphi_{1}\left(t_{n}\right) & \ldots & L_{1}\left(t_{n}, t_{n}\right)
\end{array}\right| \bar{\varphi}_{1}\left(t_{j}\right) \\
& +\int_{\left(\mathbb{R}^{d}\right)^{n}} \frac{\prod_{i} d t_{i}}{\prod_{i \neq j}\left(\omega\left(t_{i}\right)-z\right)}\left|\begin{array}{lllll}
L_{1}\left(t_{1}, t_{1}\right) & \ldots & \varphi_{1}\left(t_{1}\right) & \ldots & L_{1}\left(t_{1}, t_{n}\right) \\
L_{1}\left(t_{2}, t_{1}\right) & \ldots & \varphi_{1}\left(t_{2}\right) & \ldots & L_{1}\left(t_{2}, t_{n}\right) \\
\ldots \ldots \ldots \ldots \ldots \ldots & \ldots \ldots \ldots & \ldots \ldots & \ldots \ldots \ldots \\
L_{1}\left(t_{n}, t_{1}\right) & \ldots & \varphi_{1}\left(t_{n}\right) & \ldots & L_{1}\left(t_{n}, t_{n}\right)
\end{array}\right| \bar{\varphi}_{1}\left(t_{j}\right) .
\end{aligned}
$$

We expand the determinant in the second term in (4.5) in the entries of the $j$ th column $\left(\varphi_{1}\left(t_{1}\right), \ldots, \varphi_{1}\left(t_{n}\right)\right)^{\top}$ and obtain the sum

$$
\sum_{k=1}^{n} A_{k j} \varphi_{1}\left(t_{k}\right) \bar{\varphi}_{1}\left(t_{j}\right),
$$

where $A_{k j}$ is the cofactor of the $(k, j)$ th entry. Since $A_{j j}$, as well as the factor $\prod_{i \neq j}\left(\omega\left(t_{j}\right)-z\right)^{-1}$ multiplying the determinant, is independent of the variable $t_{j}$, we can compute the integral with respect to $t_{j}$,

$$
\int_{\mathbb{R}^{d}} \varphi_{1}\left(t_{j}\right) \bar{\varphi}_{1}\left(t_{j}\right) A_{j j} d t_{j}=A_{j j}
$$

and obviously

$$
\int_{\left(\mathbb{R}^{d}\right)^{n-1}} \frac{\prod_{i \neq j} d t_{i}}{\prod_{i \neq j}\left(\omega\left(t_{i}\right)-z\right)} A_{j j}=R_{n-1} .
$$

For the remaining integrals, we have

$$
\int_{\mathbb{R}^{d}} \varphi_{1}\left(t_{k}\right) \bar{\varphi}_{1}\left(t_{j}\right) A_{k j} d t_{j}=0 \quad \text { for } k \neq j
$$

Indeed, each term in the complete expansion of $A_{k j}$ in a sum of products of its entries contains exactly one entry of the $j$ th row, namely, $L_{1}\left(t_{j}, t_{m}\right)$. Since

$$
\int_{\mathbb{R}^{d}} L_{1}\left(t_{j}, t_{m}\right) \overline{\varphi_{1}}\left(t_{j}\right) d t_{j}=0
$$

we arrive at (4.9).

Thus, the expression on the left-hand side in (4.3) finally becomes

$$
\begin{aligned}
& R_{n}-n R_{n-1}-\left(z-\lambda_{1}\right) \\
& \quad \times \sum_{j=1}^{n} \int_{\left(\mathbb{R}^{d}\right)^{n}} \frac{\prod_{i} d t_{i}}{\prod_{i}\left(\omega\left(t_{i}\right)-z\right)}\left|\begin{array}{lllll}
L_{1}\left(t_{1}, t_{1}\right) & \ldots & \varphi_{1}\left(t_{1}\right) & \ldots & L_{1}\left(t_{1}, t_{n}\right) \\
L_{1}\left(t_{2}, t_{1}\right) & \ldots & \varphi_{1}\left(t_{2}\right) & \ldots & L_{1}\left(t_{2}, t_{n}\right) \\
\ldots \ldots \ldots \ldots \ldots \ldots & \ldots & \ldots \ldots \ldots \ldots \ldots \ldots \ldots \ldots \ldots \\
L_{1}\left(t_{n}, t_{1}\right) & \ldots & \varphi_{1}\left(t_{n}\right) & \ldots & L_{1}\left(t_{n}, t_{n}\right)
\end{array}\right| \bar{\varphi}_{1}\left(t_{j}\right) .
\end{aligned}
$$

By substituting this expression for each $n \geq 1$ into (2.3) and by taking into account the fact that $R_{0}=1$, we obtain (4.2) for $s=1$.

If formula (4.2) holds for some set $\left\{\varphi_{1}, \ldots, \varphi_{s}\right\}$ of $s$ eigenfunctions of $A$, then we supplement this set by another eigenfunction $\varphi_{s+1}$, replace $L_{s}\left(t_{1}, t_{2}\right)$ by

$$
L_{s+1}\left(t_{1}, t_{2}\right)-\left(\omega\left(t_{2}\right)-\lambda_{s+1}\right) \varphi_{s+1}\left(t_{1}\right) \bar{\varphi}_{s+1}\left(t_{2}\right)
$$

everywhere in (4.2), and, after some computations similar to those given above, arrive at the same formula (4.2) for the augmented set $\left\{\varphi_{j}\right\}_{j=1}^{s+1}$. Finally, for $s=k$ we arrive at 
the representation (3.6a), in which $v(z)$ coincides (up to the sign $(-1)^{k}$ ) with the sum $\sum_{n=k}^{\infty} \ldots$ occurring in formula (4.2) (for $s=k$ ) after the factor $\prod_{j=1}^{k}\left(\lambda_{j}-z\right)$. By the same argument as in the derivation of the estimate (2.16a) in Section 2, we readily prove that $v \in \mathcal{M}$.

The representation (3.6b) for $I^{+}(q, x)$ (under the condition $g \in \mathcal{H}$ ) can be obtained from the formula

$$
\begin{aligned}
& I(q, z)=\prod_{j=1}^{k}\left(\lambda_{j}-z\right) \sum_{n=k}^{\infty} \frac{1}{n !} \int_{\left(\mathbb{R}^{d}\right)^{(n+2)}} \frac{D(q, p) g\left(t_{0}\right) \prod_{i=0}^{n} d t_{i} d p}{(\omega(p)-z) \prod_{i=0}^{n}\left(\omega\left(t_{i}\right)-z\right)} \\
& \times \sum_{j_{1}, \ldots, j_{k}}\left|\begin{array}{ccccccc}
L_{k}(p, p) & \ldots & \varphi_{1}(p) & \ldots & \varphi_{k}(p) & \ldots & L_{k}\left(p, t_{n}\right) \\
L_{k}\left(t_{0}, p\right) & \ldots & \varphi_{1}\left(t_{0}\right) & \ldots & \varphi_{k}\left(t_{0}\right) & \ldots & L_{k}\left(t_{0}, t_{n}\right) \\
L_{k}\left(t_{1}, p\right) & \ldots & \varphi_{1}\left(t_{1}\right) & \ldots & \varphi_{k}\left(t_{1}\right) & \ldots & L_{k}\left(t_{1}, t_{n}\right) \\
\ldots \ldots & \ldots & \ldots & \ldots & \ldots & \ldots & \ldots \\
L_{k}\left(t_{n}, p\right) & \ldots & \varphi_{1}\left(t_{n}\right) & \ldots & \varphi_{k}\left(t_{n}\right) & \ldots & L_{k}\left(t_{n}, t_{n}\right)
\end{array}\right| \prod_{m=1}^{k} \bar{\varphi}_{m}\left(t_{j_{m}}\right)
\end{aligned}
$$

where the sum $\sum_{j_{1}, \ldots, j_{k}}$ is taken over all arrangements of $k$ columns in $n+2$ places.

Thus, we arrive at the representation (3.6b), where the function $I^{\prime}(q, z)$ coincides (up to the sign $\left.(-1)^{k}\right)$ with the sum occurring after the product $\prod_{j=1}^{k}\left(\lambda_{j}-z\right)$ in (4.10). Just as in the case of the function $v$, one can prove that $I^{\prime}(q, x)$ satisfies an estimate similar to (2.16a) and hence belongs to the ideal $\mathcal{M}$ for each $q$. The proof of Lemma 3 is complete.

\section{Appendix A}

To obtain the representation (2.18), note that the inner integral in (2.17) is the Fourier transform of the product

$$
M_{n}\left(x_{1}, \ldots, x_{n}\right) \cdot \prod_{j=1}^{n} \delta\left(x-x_{j}\right)
$$

where

$$
M_{n}\left(x_{1}, \ldots, x_{n}\right)=\int_{\left(\mathbb{R}^{d}\right)^{n}} \frac{G_{n}\left(s_{1}, \ldots, s_{n}\right)}{\prod_{j}\left(\omega\left(s_{j}\right)-x_{j}-i 0\right)} \prod_{j} d s_{j} .
$$

Hence this transform is equal to the convolution

$$
\int_{\left(\mathbb{R}^{1}\right)^{n}} \widetilde{M}_{n}\left(\tau_{1}, \ldots, \tau_{n}\right) \prod_{j} e^{-i\left(t_{j}-\tau_{j}\right) x} \prod_{j} d \tau_{j},
$$

where

$$
\begin{aligned}
\widetilde{M}_{n}\left(\tau_{1}, \ldots, \tau_{n}\right) & =\int_{\left(\mathbb{R}^{1}\right)^{n}} \prod_{j} e^{-i \tau_{j} x_{j}} \prod_{j} d x_{j} \int_{\left(\mathbb{R}^{d}\right)^{n}} \prod_{j} d s_{j} \frac{G_{n}\left(s_{1}, \ldots, s_{n}\right)}{\prod_{j}\left(\omega\left(s_{j}\right)-x_{j}-i 0\right)} \\
& =\prod_{j=1}^{n}\left(2 \pi i \theta\left(\tau_{j}\right)\right) \int_{\left(\mathbb{R}^{d}\right)^{n}} \prod_{j=1}^{n} e^{-i \tau_{j} \omega\left(s_{j}\right)} G_{n}\left(s_{1}, \ldots, s_{n}\right) \prod_{j=1}^{n} d s_{j}=S_{n}\left(\tau_{1}, \ldots, \tau_{n}\right) .
\end{aligned}
$$

Here we have used the identity 4 ]

$$
\int_{-\infty}^{\infty} \frac{e^{-i \tau x}}{\omega(s)-x-i 0} d x=2 \pi i \theta(\tau) e^{-i \tau \omega(s)}
$$

By integrating (A.1) with respect to $x$ and by using the identity

$$
\int_{-\infty}^{\infty} e^{-i\left(\sum_{j} t_{j}-\sum_{j} \tau_{j}\right) x} d x=2 \pi \delta\left(t-\sum_{j} \tau_{j}\right),
$$

we arrive at (2.18). 


\section{Appendix B}

Here we present a derivation of the estimate (2.19).

First, consider the integral

$$
\int_{\mathbb{R}^{d}} e^{-i \tau \omega(s)} f(s) d s
$$

where $f \in S\left(\mathbb{R}^{d}\right)$. By the argument usually invoked when finding the asymptotics of oscillatory integrals by the stationary phase method (e.g., see [12]), we find that the integral (B.1) admits the estimate

$$
\begin{aligned}
\left|\int_{\mathbb{R}^{d}} e^{-i \tau \omega(s)} f(s) d s\right| \leq \frac{1}{(|\tau|+1)^{d / 2}} & \left(B_{1}|f(0)|+B_{2} \max _{s,|\alpha| \leq m}\left|\left(\partial^{\alpha} f\right)(s)\right| \cdot \frac{1}{|\tau|+1}\right. \\
& \left.+B_{3} \int_{\mathbb{R}^{d}|\alpha| \leq\left[\frac{d}{2}\right]+1} \max _{\mid}\left|\left(\partial^{\alpha} f\right)(s)\right| d s \cdot \frac{1}{(|\tau|+1)^{1 / 2}}\right) .
\end{aligned}
$$

Here $B_{1}>0, B_{2}>0$, and $B_{3}>0$ are constants depending on the function $\omega, m$ is an absolute constant, $|\alpha|=\max _{i} \alpha_{i}$, and $\alpha=\left(\alpha_{1}, \ldots, \alpha_{d}\right)$.

In a similar way, we estimate the multiple oscillatory integral

$$
I_{n}\left(\left\{\tau_{j}\right\}_{1}^{n}\right)=\int_{\left(\mathbb{R}^{d}\right)^{n}} G_{n}\left(s_{1}, \ldots, s_{n}\right) \prod_{j} e^{-i \tau_{j} \omega\left(s_{j}\right)} \prod_{j} d s_{j} .
$$

Namely,

$$
\begin{aligned}
& I_{n}\left(\left\{\tau_{j}\right\}_{1}^{n}\right) \leq \frac{1}{\prod_{j}\left(\left|\tau_{j}\right|+1\right)^{d / 2}} \sum_{\substack{\theta_{1}, \theta_{2}, \theta_{3} \\
\theta_{1} \sqcup \theta_{2} \sqcup \theta_{3}=\{1, \ldots, n\}}} B_{1}^{\left|\theta_{1}\right|} B_{2}^{\left|\theta_{2}\right|} B_{3}^{\left|\theta_{3}\right|} \int_{\left(\mathbb{R}^{d}\right)^{\left|\theta_{3}\right|}} \prod_{j \in \theta_{3}} d s_{j} \\
& \times \max _{\substack{s_{j},\left|\alpha_{j}\right| \leq m, j \in \theta_{2} \\
\left|\bar{\alpha}_{j}\right| \leq\left[\frac{d}{2}\right]+1, j \in \theta_{3}}}\left|\prod_{j \in \theta_{3}} \partial_{s_{j}}^{\bar{\alpha}_{j}} \prod_{j \in \theta_{2}} \partial_{s_{j}}^{\alpha_{j}} G_{n}\left(\left\{s_{j}=0, j \in \theta_{1}\right\},\left\{s_{j}, j \in \theta_{2} \sqcup \theta_{3}\right\}\right)\right| \\
& \times \prod_{j \in \theta_{2}} \frac{1}{\left(\left|\tau_{j}\right|+1\right)} \prod_{j \in \theta_{3}} \frac{1}{\left(\left|\tau_{j}\right|+1\right)^{1 / 2}} .
\end{aligned}
$$

Here the sum $\sum_{\theta_{1}, \theta_{2}, \theta_{3}}$ is taken over all partitions of the indexing set $\{1, \ldots, n\}$ into three subsets $\theta_{i}, i=1,2,3$ (possibly, empty). Note that since the determinant $G_{n}\left(s_{1}, \ldots, s_{n}\right)$ vanishes whenever two of the variables coincide, it follows that $\left|\theta_{1}\right| \leq 1$.

We introduce the function

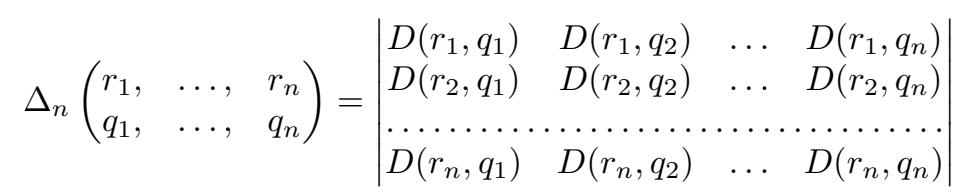

of $2 n$ variables $\left(r_{1}, \ldots, r_{n}\right)$ and $\left(q_{1}, \ldots, q_{n}\right)$. Obviously,

$$
G_{n}\left(s_{1}, \ldots, s_{n}\right)=\left.\Delta_{n}\left(\begin{array}{lll}
r_{1}, & \ldots, & r_{n} \\
q_{1}, & \ldots, & q_{n}
\end{array}\right)\right|_{r_{j}=q_{j}=s_{j}, j=\overline{1, n}} .
$$

If $f(r, q)$ is a function of two variables $r, q \in \mathbb{R}^{d}$ and $F(s)=\left.f(r, q)\right|_{r=q=s}$, then

$$
\left(\partial_{s}^{\alpha} F\right)(s)=\left.\sum_{\beta+\gamma=\alpha} \frac{1}{\beta !} \frac{1}{\gamma !}\left(\partial_{r}^{\beta} \partial_{q}^{\gamma} f\right)(r, q)\right|_{r=q=s},
$$


where

$$
\alpha !=\prod_{i=1}^{d} \alpha_{i} !
$$

for any multi-index $\alpha=\left(\alpha_{1}, \ldots, \alpha_{d}\right)$.

Thus,

$$
\begin{aligned}
& \left(\prod_{j \in \theta_{3}} \partial_{s_{j}}^{\bar{\alpha}_{j}} \prod_{j \in \theta_{2}} \partial_{s_{j}}^{\alpha_{j}} G_{n}\right)\left(\left\{s_{j}=0, j \in \theta_{1}\right\},\left\{s_{j}, j \in \theta_{2} \sqcup \theta_{3}\right\}\right) \\
& =\prod_{j \in \theta_{3}} \sum_{\bar{\beta}_{j}+\bar{\gamma}_{j}=\bar{\alpha}_{j}} \frac{1}{\bar{\beta}_{j} ! \bar{\gamma}_{j} !} \prod_{j \in \theta_{2}} \sum_{\beta_{j}+\gamma_{j}=\alpha_{j}} \frac{1}{\beta_{j} ! \gamma_{j} !}
\end{aligned}
$$

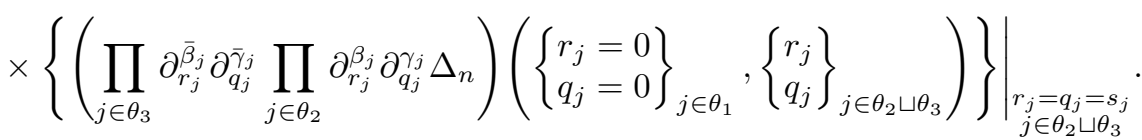

The expression in braces is the determinant of the matrix $\Gamma=\left(\Gamma_{j, j^{\prime}}\right)_{j, j^{\prime}=\overline{1, n}}$ whose entries are given in the following table:

\begin{tabular}{|c|c|c|c|}
\hline$\Gamma_{j, j^{\prime}}$ & $j^{\prime} \in \theta_{1}$ & $j^{\prime} \in \theta_{2}$ & $j^{\prime} \in \theta_{3}$ \\
\hline$j \in \theta_{1}$ & $D(0,0)$ & $\left(\partial_{q}^{\gamma_{j^{\prime}}} D\right)\left(0, q_{j^{\prime}}\right)$ & $\left(\partial_{q}^{\bar{\gamma}_{j^{\prime}}} D\right)\left(0, q_{j^{\prime}}\right)$ \\
\hline$j \in \theta_{2}$ & $\left(\partial_{r}^{\beta_{j}} D\right)\left(r_{j}, 0\right)$ & $\left(\partial_{r}^{\beta_{j}} \partial_{q}^{\gamma_{j^{\prime}}} D\right)\left(r_{j}, q_{j^{\prime}}\right)$ & $\left(\partial_{r}^{\beta_{j}} \partial_{q}^{\bar{\gamma}_{j^{\prime}}} D\right)\left(r_{j}, q_{j^{\prime}}\right)$ \\
\hline$j \in \theta_{3}$ & $\left(\partial_{r}^{\bar{\beta}_{j}} D\right)\left(r_{j}, 0\right)$ & $\left(\partial_{r}^{\bar{\beta}_{j}} \partial_{q}^{\gamma_{j^{\prime}}} D\right)\left(r_{j}, q_{j^{\prime}}\right)$ & $\left(\partial_{r}^{\bar{\beta}_{j}} \partial_{q}^{\bar{\gamma}_{j^{\prime}}} D\right)\left(r_{j}, q_{j^{\prime}}\right)$ \\
\hline
\end{tabular}

Set

$$
\max _{\substack{r, q \in \mathbb{R}^{d} \\ \beta,|\beta|<\varkappa ; \gamma,|\gamma|<\varkappa}}\left|\left(\partial_{r}^{\beta} \partial_{q}^{\gamma} D\right)(r, q)\right|=R, \quad \max _{\substack{r, q \in \mathbb{R}^{d} \\ \beta,|\beta|<\varkappa ; \gamma,|\gamma|<\varkappa}}\left|\left(\partial_{r}^{\beta} \partial_{q}^{\gamma} D\right)(r, q)\left(q^{2}+1\right)^{d}\right|=S,
$$

where $\varkappa=\max \{m,[d / 2]+1\}$.

By using Hadamard's inequality [9], we obtain

$$
|\operatorname{det} \Gamma| \leq R^{\left|\theta_{1}\right|+\left|\theta_{2}\right|} S^{\left|\theta_{3}\right|} n^{n / 2} \prod_{j \in \theta_{3}} \frac{1}{\left(q_{j}^{2}+1\right)^{d}} .
$$

Since

$$
\sum_{\beta+\gamma=\alpha} \frac{1}{\beta ! \gamma !}=\frac{1}{\alpha !} \sum_{\beta+\gamma=\alpha} \frac{\alpha !}{\beta ! \gamma !}=\frac{1}{\alpha !} 2^{\alpha_{1}+\ldots+\alpha_{d}} \leq 2^{d}
$$

we find that the absolute value of the expression (B.4) does not exceed

$$
2^{d\left(\left|\theta_{2}\right|+\left|\theta_{3}\right|\right)} R^{\left|\theta_{1}\right|+\left|\theta_{2}\right|} S^{\left|\theta_{3}\right|} n^{n / 2} \prod_{j \in \theta_{3}} \frac{1}{\left(q_{j}^{2}+1\right)^{d}} .
$$

By substituting this estimate into (B.3) and by integrating over the variables $\left\{s_{j}=q_{j}\right.$, $\left.j \in \theta_{3}\right\}$, we obtain

$$
\begin{aligned}
I_{n}\left(\left\{\tau_{j}\right\}_{1}^{n}\right) & \leq \frac{n^{n / 2}}{\prod_{j}\left(\left|\tau_{j}\right|+1\right)^{d / 2}} \sum_{\substack{\theta_{1}, \theta_{2}, \theta_{3} \\
\theta_{1} \sqcup \theta_{2} \sqcup \theta_{3}=\{1, \ldots, n\}}} B_{1}^{\left|\theta_{1}\right|} B_{2}^{\left|\theta_{2}\right|} B_{3}^{\left|\theta_{3}\right|} 2^{d\left(\left|\theta_{2}\right|+\left|\theta_{3}\right|\right)} R^{\left|\theta_{1}\right|+\left|\theta_{2}\right|} S^{\left|\theta_{3}\right|} T^{\left|\theta_{3}\right|} \\
& \leq \frac{n^{n / 2}}{\prod_{j}\left(\left|\tau_{j}\right|+1\right)^{d / 2}} \cdot \widehat{C}^{n} \cdot\left(\text { the number of partitions }\left(\theta_{1}, \theta_{2}, \theta_{3}\right)\right) .
\end{aligned}
$$


Here

$$
T=\int_{\mathbb{R}^{d}} \frac{d s}{\left(s^{2}+1\right)^{d}} \quad \text { and } \quad \widehat{C}=\max \left\{B_{1}, B_{2}, B_{3}, 2^{d} R, S T\right\} .
$$

Since the number of partitions $\left(\theta_{1}, \theta_{2}, \theta_{3}\right)$ of the set $\{1, \ldots, n\}$ does not exceed $3^{n}$, we arrive at the estimate

$$
I_{n}\left(\left\{\tau_{j}\right\}_{1}^{n}\right) \leq \frac{n^{n / 2}}{\prod_{j=1}^{n}\left(\left|\tau_{j}\right|+1\right)^{d / 2}} \cdot(3 \widehat{C})^{n},
$$

which gives (2.19).

\section{REFERENCES}

[1] È. R. Akchurin and R. A. Minlos, A new method in the scattering theory, Vestnik Moskov. Univ. Ser. I Mat. Mekh. 65 (2010), no. 6, 27-32; English transl., Moscow Univ. Math. Bull. 65 (2010), no. 6, 247-251. MR2814987

[2] L. D. Faddeev, On the Friedrichs model in the theory of perturbations of a continuous spectrum, Trudy Mat. Inst. Steklov 73 (1964), 292-313; English transl., Amer. Math. Soc., Transl. Ser. 2 , vol. 62, Amer. Math. Soc., Providence, RI, 1967, pp. 177-203. MR0178362 (31:2620)

[3] D. R. Yafaev, Mathematical scattering theory: General theory, Izdat. Sankt-Peterburg. Univ., St. Petersburg, 1994; English transl., Transl. of Math. Monographs, vol. 105, Amer. Math. Soc., Providence, RI, 1992 (reprinted with corrections in 1998). MR1180965 (94f:47012)

[4] I. M. Gelfand and G. E. Shilov, Generalized functions. Vol. 1. Properties and operations, Gostekhizdat, Moscow, 1959; English transl., Academic Press, New York-London, 1964. MR0435831 $(55: 8786 \mathrm{a})$

[5] I. M. Gelfand and G. E. Shilov, Generalized functions. Vol. 2. Spaces of fundamental and generalized functions, Gostekhizdat, Moscow, 1958; English transl., Academic Press, New York-London, 1968. MR 0230128 (37:5693)

[6] M. Reed and B. Simon, Methods of modern mathematical physics. III. Scattering theory, Academic Press, New York-London, 1979. MR.529429 (80m:81085)

[7] F. Riesz and B. Sz.-Nagy, Leçons d'analyse fonctionnelle, Gauthier-Villars, Paris; copublished with Akadémiai Kiadó, Budapest, 1965. (French) MR0179567 (31:3815)

[8] A. N. Kolmogorov and S. V. Fomin, Elements of the theory of functions and functional analysis, 3rd ed., Nauka, Moscow, 1972; English transl. of the 2nd ed. (1968), Prentice-Hall, Englewood Cliffs, NJ, 1970. MR0234241 (38:2559)

[9] W. V. Lovitt, Linear integral equations, Dover, New York, 1950.

[10] N. I. Muskhelishvili, Singular integral equations, Nauka, Moscow, 1968; English transl. of the 2nd ed. (1962), Wolters-Noordhoff, Groningen, 1967. MR0355495 (50:7969)

[11] I. I. Privalov, Boundary properties of analytic functions, 2nd ed., Gosudarstv. Izdat. Tekhn.-Teor. Lit., Moscow, 1950. (Russian) MR0047765 (13:926h)

[12] M. V. Fedoryuk, Asymptotics. Integrals and series, Nauka, Moscow, 1987. MR950167 (89j:41045)

[13] È. R. Akchurin and R. A. Minlos, Scattering theory for a class of two-particle operators of mathematical physics (the case of weak interaction), Izv. Ross. Akad. Nauk Ser. Mat. (to appear)

[14] I. M. Gelfand, D. A. Raikov, and G. E. Shilov, Commutative normed rings, Gosudarstv. Izdat. Fiz.Mat. Lit., Moscow, 1960; English transl., Chelsea Publishing Co., New York, 1964. MR0123921 (23:A1242)

[15] V. I. Paraska, On asymptotics of eigenvalues and singular numbers of linear operators which increase smoothness, Mat. Sb. 68 (110) (1965), no. 4, 623-631. (Russian) MR0199749(33:7892)

Mechanics and Mathematics Faculty, Moscow State University, Moscow 119991, Russian FEDERATION

E-mail address: eakchurin@gmail.com

Institute for Information Transmission Problems, Moscow 127994, Russian Federation

E-mail address: minl@iitp.ru 